follow-up strategies tend to shift towards personalised care with focus on patient education and self-management. There are limited data on gynaecological cancer survivors' self-management abilities. This study aimed to assess the relationship between self-management skills and adherence to current follow-up guidelines among gynaecological cancer survivors in the Netherlands, Norway, and Denmark. We also assessed the relationship between adherence to follow-up and use of additional healthcare services.

Methodology We conducted an international, multi-centre, cross-sectional study. Gynaecological cancer survivors were recruited 1-5 years after completion of treatment. We obtained information on clinical characteristics, demographics, self-management (Health Education Impact Questionnaire), follow-up visits, and use of healthcare services by validated questionnaires. Based on national guidelines, we categorised participants as adherent if they attended the recommended number of follow-up visits, non-adherent if they had fewer visits than recommended, or over-users if they had more visits than recommended.

Result(s)* Of 4455 invited survivors, 2428(55\%) returned the questionnaires. After excluding 992 randomly selected Danish survivors to even the distribution between countries and survivors with more than 5 years follow-up, 911 survivors were included in the study. Survivors with high selfmanagement skills (e.g. ability to manage medical and psychosocial aspects of one's illness) most frequently attended the recommended number of follow-up visits. There were lower self-management skills in the health-directed activity domain, indicating less health-promoting physical activity (OR 1.54, 95\% CI 1.03-2.32) among non-adherent survivors compared to adherent survivors. No other associations between self-management and follow-up adherence were revealed. Non-adherent survivors were likely to be older, treated by surgery only, Danish residents and have endometrial cancer. Over-users not only reported more follow-up visits but also used additional healthcare services, such as oncologic rehabilitation and/or oncological nurses, more frequently than adherent survivors.

Conclusion* Possession of low self-management skills appears to reduce the likelihood of adhering to national guidelines for gynaecological cancer follow-up. As personalisation of care is gradually implemented, a special attention on patient education for survivors at risk of low self-management may help to ensure their adherence to recommended follow-up.

\section{UNDERSTANDING THE RECOVERY PROCESS AND RETURN TO DAILY ACTIVITY IN WOMEN TREATED FOR CERVICAL CANCER}

${ }^{1} \mathrm{~N}$ Millet, ${ }^{2} \mathrm{E}$ Moss*, ${ }^{1} \mathrm{~F}$ Munir, ${ }^{1} \mathrm{H}$ Mcdermott. ' ${ }^{1}$ Loughborough University, School of Sport, Exercise and Health Sciences, Loughborough, UK; ${ }^{2}$ University of Leicester, Leicester Cancer Research Centre, Leicester, UK

\subsection{6/ijgc-2021-ESG0.574}

Introduction/Background* Cervical cancer is predominantly a cancer of younger women and improvements in oncological outcomes have resulted in more women surviving cervical cancer and living with the long-term effects of treatment at a younger age. Understanding the recovery process and the experience of cancer survivors is essential to increase awareness of the long-term psychological and physical needs of survivors.

Methodology A qualitative study was conducted with the aim of understanding the recovery process and return to daily activity in women treated for cervical cancer. Interviews were undertaken face-to-face and via the telephone using a semistructured interview schedule. Inductive thematic analysis was used to derive a thematic framework from the data.

Result(s)* Twenty-one women who had been treated for cervical cancer aged 18-60 years and were living in the UK participated in an interview. Analysis of the data revealed themes which described participants' experience and perceptions of treatment as a paradox; physical recovery and selfmanagement strategies of treatment related issues; Psychological coping both in the short and long-term after treatment. A key finding were the nuances in different experiences between treatment types, with physical changes perceived to be more disruptive following radical treatments, whilst psychological repercussions seemed to be significant regardless of treatment type.

Conclusion* This study provides novel insight into the varied recovery experiences of those treated with surgery, chemotherapy, and radiotherapy after cervical cancer, which can be used to improve recovery experiences for future survivors.

\section{CREATSAS VAGINOPLASTY IN PATIENTS AFTER PELVIC CHEMORADIATION IN CERVICAL CANCER: A PRELIMINARY STUDY}

B Segarra Vidal ${ }^{*}$, P Padilla Iserte, V Lago, MT Luis Javier, S Domingo. Hospital Universitario y Politécnico de La Fe, Gynecological oncology Unit, València, Spain

\subsection{6/ijgc-2021-ESGO.575}

Introduction/Background* Chemoradiotherapy followed by brachytherapy is the standard treatment in locally advanced cervical cancer and vaginal stenosis is one of the most common side effects after pelvic radiation. Sexual quality is the main condition that does not improve over time in these patients.

Methodology The main objective of this study was to assess the impact of the Creatsas vaginoplasty on the improvement in the sexual life of patients with cervical cancer who have received chemoradiotherapy and have sexual dysfunction measured by The Female Sexual Function Index. The design was an observational prospective individual cohort study between January 2018 - December 2019 of patients report outcomes (PRO) using The Female Sexual Function Index.

Result(s)* A total of 12 patients were evaluated and 6 patients met the inclusion criteria. Among all patients, the global scores of The Female Sexual Function Index questionnaire improved after surgery except for one patient. No patients had post-operative complications. The median of the global score before the surgery was 22.95 (IQR 19.18, 24.92), after 6 months was 26.95 (IQR 24.95, 27.23) and, after 12 months 24.5 (IQR 23.18, 26.42). The global score had increased after the surgery $(\mathrm{OR}=1.27 \mathrm{CI} 95 \%[1.087 ; 1.499])$.

Conclusion* This preliminary study suggests that the Creatsas procedure improves the sexual life of cervical cancer patients with vaginal stenosis who have received chemoradiotherapy as the first treatment. 